\title{
The Method for Measuring the Loop Resistance and the Temperature Rise Calculation of GIS Conductor Pole
}

\author{
Li Zhou*, Tiecheng Lu, Bo Zhang \\ School of Electrical Engineering, Wuhan University, Wuhan, China \\ Email: ${ }^{*}$ 2010282070205@whu.edu.cn
}

Received February 2015

\begin{abstract}
This paper studies the method for measuring the loop resistance of GIS conductor pole based on the super capacitor producing impulse current up to several thousand amperes. This method overcomes the limitations of conventional diagnostic method. Typical GIS conductor poles are chosen. Based on FEA and lab tests, the effect of different forms of current and contact condition, relationship between the temperature of contact and the loop resistance is researched. In fullscale testing under realistic operating conditions on the new $220 \mathrm{kV}$ GIS using prototype instrumentation a very good sensitivity in an early stage was obtained.
\end{abstract}

\section{Keywords}

Loop Resistance, Gas-Insulated Substations (GIS), Impulse Current, Finite Element Analysis (FEA)

\section{Introduction}

In the operation of power system, gas insulated substation (GIS) conductor pole insertion depth is not enough under the procession of installation, thus the surface of contact produces oxide film and burr. It causes the loop resistance of GIS conductor pole increases and leads to the temperature of contact increases. In addition, the temperature of contact increases causes the loop resistance of GIS conductor pole further increases. When case gets serious, it will influence the operated safety of power system. So accurately measuring the loop resistance of GIS is necessary.

Although the conventional diagnostic method, such as the method of dc voltage drop has stable current and can greatly simplifies the resistance measurements. It rated for kiloamperes tend to become very bulky and expensive, so dc excitation is not found to be a viableoption [1]-[5].

The studying method for measuring the loop resistance of GIS conductor pole is based on the super capacitor produce kiloamperes impulse current. Similar to four-point resistance measurements, the method relies on testing passing electric currents through the section and recording the associated voltage drops. The feasibility of method and the accuracy of results are verified by experiment [6]-[9].

Based on the analysis of typical GIS conductor pole, a thermal model of contactor is proposed in this paper.

\footnotetext{
${ }^{*}$ Corresponding author.
}

How to cite this paper: Zhou, L., Lu, T.C. and Zhang, B. (2015) The Method for Measuring the Loop Resistance and the Temperature Rise Calculation of GIS Conductor Pole. Journal of Power and Energy Engineering, 3, $306-312$. 
The temperature distribution of the GIS conductor pole was achieved by FEM simulation and the results were proved by experiments. The effect of the different forms of current and different contact condition of contact on the temperature of contact are researched. Furthermore, the relationship between the temperature of contact surface and the loop resistance of GIS conductor pole are analyzed.

\section{Resistance Measurement}

The proposed method for measuring the loop resistance of GIS conductor pole is based on the impulse current. The super capacitor, standard resistance and tests components constitute second-order circuit of non oscillation and produce kA-level impulse current. The peak current and the corresponding voltage drop of the GIS conductor pole are used to get the loop resistance, in order to eliminate the impact of the inductance on the measurement result.

The relationship between voltage drop and current across the GIS section are determined by the Equation (1)

$$
u(t)=R(i, t) i(t)+L \frac{\mathrm{d} i(t)}{\mathrm{d} t} .
$$

where $R$ and $L$ are the resistance and inductance, respectively, of the part of the circuit between the voltage probes, see Figure 1.

As can be seen from Figure 2, the derivative of the current is large and positive in the first milliseconds, the inductive part of voltage drop is significantly greater than the resistive part. When the current has its maximum value, $\mathrm{di} / \mathrm{d} t$ equals zero and the voltage drop is purely resistive.

$$
\frac{u(n)}{i(n)}=R(n)+L \frac{i(n+1)-i(n)}{i(n) \Delta t} .
$$

where $C$ represents the super capacitor, $R$ is the total circuit resistance including super capacitor resistance, wire

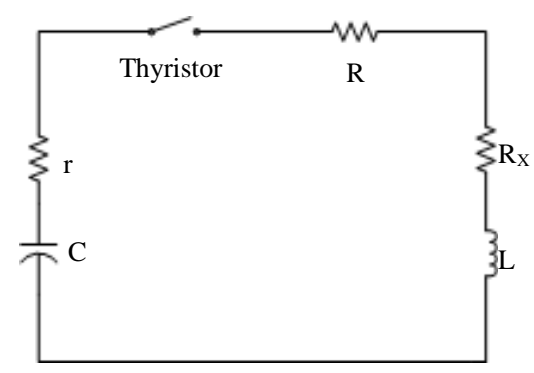

Figure 1. The main discharge circuit equivalent circuit.

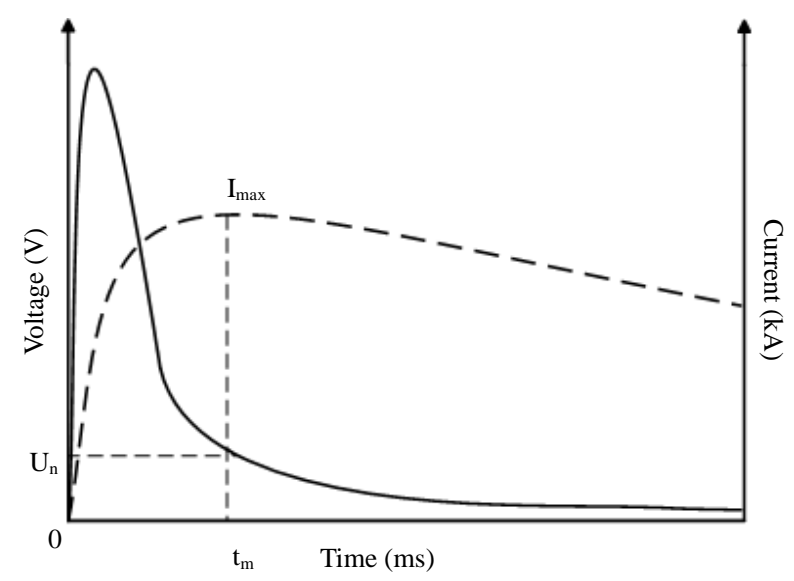

Figure 2. Voltage drop measured across a GIS section (solid line) when a currentpulse (broken line) is passed through. 
resistance, shunt resistance and the measured GIS conductive pole loop resistance, and $L$ is inductance of GIS conductor pole.

Having determined $L$, the inductive part of the voltage drop is calculated and then subtracted from $u(n)$ for all $n$. By dividing this result with $i(n)$, a time series $R(n)$ being the resistance of the GIS section, is found.

$R(n)$ is, however, not constant, due to variations in the current penetration depth. In order to avoid this source of error, the resistance value from a measurement is always taken as the value found at the same point of the current pulse, namely, as the current pulse is at its peak value.

Thus, a section of a GIS is examined by passing current pulses of different magnitudes, calculating the resistance when the current pulse is at its peak value each time. And as described earlier, if the resistance varies, this indicates that one or more of the contacts has too high of a resistance.

\section{Modeling and Simulations}

\subsection{Physics of Heat Sources}

In the FEM thermal-electrical coupled simulation analysis, make the following assumptions to facilitate the calculation [10]:

In order to find the electrical resistance at the main contacts, the radius $a_{\varepsilon}$ of the elastically deformed contact area is expressed by

$$
a_{\varepsilon}=\sqrt[3]{\frac{3}{4} F_{k}\left(\frac{1-\mu_{1}^{2}}{E_{1}}+\frac{1-\mu_{2}^{2}}{E_{2}}\right)\left(\frac{1}{r_{1}}+\frac{1}{r_{2}}\right)^{-1}} .
$$

where $F_{k}$ is the contact force, $E_{i}$ and $\mu_{i}$ are the moduli of elasticity and the Poisson's ratios, respectively, and $r_{i}$ are radii of the spherical members in contact. The radius $a_{\varepsilon}$ is then used to calculate the contact resistance

$$
R_{k}=\frac{\bar{\rho}}{2 a_{\varepsilon}} .
$$

According to Holm's ellipsoid model, the average specific electrical resistivity of the two different materials in contact is denoted by $\bar{\rho}$.

It is known that the impact of heating is considered in the resistance changes. Therefore the temperature dependent term: where $\alpha$ is coefficient of resistivity $\Delta T$ is temperature difference.

$$
R_{k}=R_{k o}\left(1+\frac{2}{3} \alpha \Delta T\right) \text {. }
$$

An applied current flowing in the electrically conducting path causes Joule heat $P=R I^{2}$ to be generated. This heat increases the temperature of the conducting parts. Due to the resulting temperature differences, heat is transported by means of conduction, convection and radiation.

\subsection{Simulation Model}

Choose typical spring conductive connector as simulation model, it contacts by spring deformation on every point of contact pressure, ensure the contact well. As shown in Figure 3(a), where the material of No.1-No.3 and No.5 is aluminum, the material of No.4 is copper, and the material of No.6 is insulating. In this model, completely insertion depth is $55 \mathrm{~mm}$, as shown in Figure 3(b).

\subsection{Result of Simulation}

- Different forms of current

Through the formula calculate the contact resistance of GIS conductor pole that contact well. According to the basis of the experiment measured the impulse current (current amplitude is 2424A, time is $1 \mathrm{~s}$ ). In the same conditions of current amplitude and duration, GIS conductor pole can be used for thermal simulation. Figure 4 shows the temperature is effected of dc slightly greater than the impact current. 
- Different contact condition

Through the formula calculate the contact resistance of different contact condition. Under the conditions of impulse current, GIS conductor pole can be used for thermal simulation. Figure $\mathbf{5}$ shows the temperature is higher when the contact condition is worse.

\section{Experiment Tests}

\subsection{Laboratory Tests}

Table 1 shows different test methods of measuring GIS conductor pole that contact well. Two methods of testing have the stability of test results. What's more the results are basically identical. It is means that two methods of measuring are accurate. Furthermore, the result of temperature is a little influence, due to the amplitude of dc is

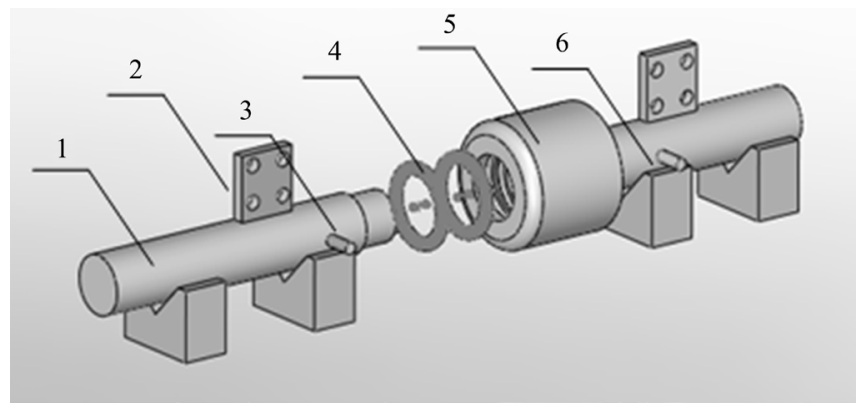

(a)

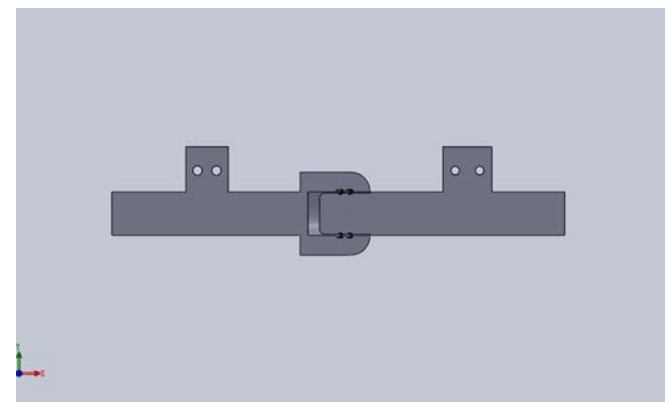

(b)

Figure 3. Typical GIS conductor pole model. (a) Physical model; (b) Simulation model.

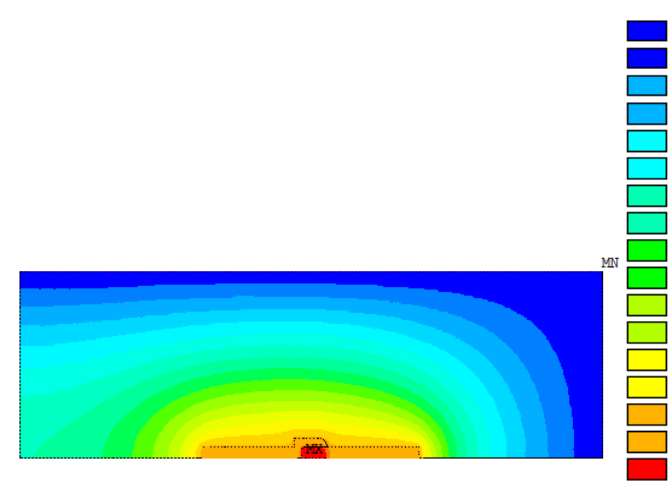

(a)

\begin{tabular}{ll}
\hline$\square$ & 20 \\
20.063 \\
20.125 \\
20.188 \\
\hline$\square$ \\
20.25 \\
20.313 \\
\hline$\square$ \\
20.376 \\
20.438 \\
20.501 \\
\hline$\square$ \\
\hline$\square$ \\
20.564 \\
\hline$\square$ \\
20.626 \\
20.759 \\
\hline$\square$ \\
20.814 \\
20.877 \\
20.939 \\
21.002
\end{tabular}

21.002

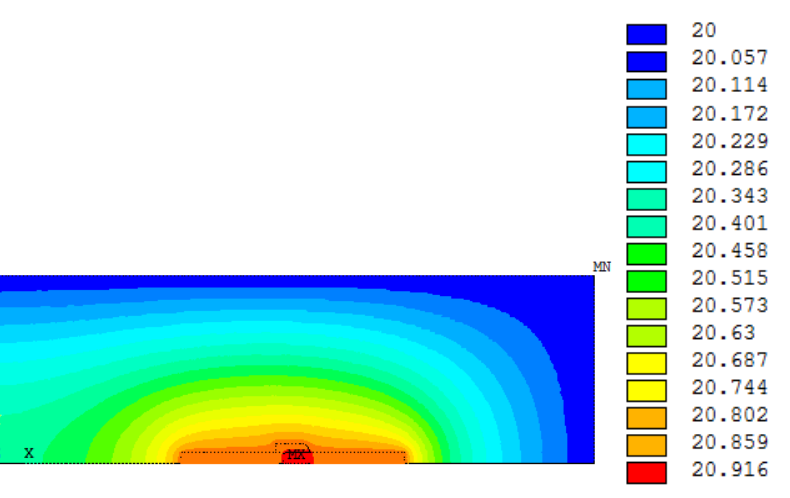

(b)

Figure 4. Calculation results of the temperature of different forms of current. (a) Current source is dc; (b) Current source is impulse current.

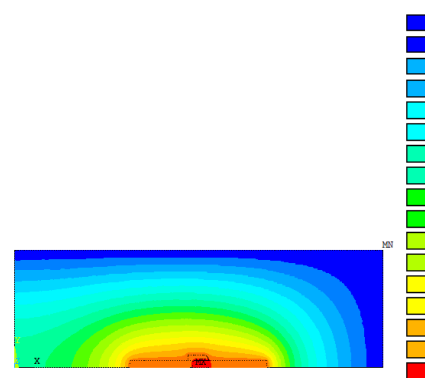

(a)

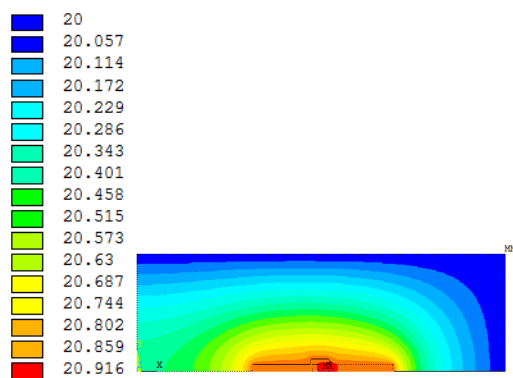

(b)

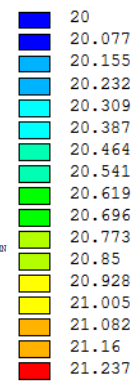

21.237

Figure 5. Calculation results of the temperature of different contact condition. (a) The contact resistance is $10 \mu \Omega$; (b) The contact resistance is $15 \mu \Omega$; (c) the contact resistance is $20 \mu \Omega$. 
not large; as well as the amplitude of impact current is large but the time of duration is short, so as the temperature is. The results also proved the correctness of the simulation calculation.

Table 2 shows different test methods of measuring GIS conductor pole under different contact conditions. The result of dc voltage drop haven't changed much. It shows that under the condition is bad; the conventional diagnostic method can't find the defect of GIS conductor pole. However, the result of the contact resistance increases along with the rising of the test current in the method of impact current voltage drop. It means that these methods can response the contact condition of GIS conductor pole effectively.

\subsection{Full-Scale Tests}

During the comprehensive laboratory measurements as well as full-scale tests under realistic conditions on GISs installed in $220 \mathrm{kV}$ grids, a few results will be presented in this section.

Table 3 shows resistance values measured on a new $220 \mathrm{kV}$ GIS by applying current pulses with peak values up to around $3.5 \mathrm{kA}$. The resistances in this section are the same for all currents within standard deviations of $0.5 \%$. Hence the contacts in this section show no signs of aging.

Table 1. Different test method of measuring resistance.

\begin{tabular}{cccc}
\hline & current source is dc & \multicolumn{2}{c}{ current source is impulse current } \\
\hline current (A) & resistance $(\mu \Omega)$ & current $(\mathrm{A})$ & resistance $(\mu \Omega)$ \\
\hline 100 & 16.103 & 1000 & 15.988 \\
200 & 16.159 & 1500 & 15.992 \\
300 & 16.235 & 2000 & 15.919 \\
400 & 16.278 & 2500 & 16.004 \\
500 & 16.304 & 3000 & 16.064 \\
\hline
\end{tabular}

Table 2. Different test method of measuring GIS conductor pole under different contact condition.

\begin{tabular}{cccc}
\hline \multicolumn{2}{c}{ current source is dc } & \multicolumn{2}{c}{ current source is impulse current } \\
\hline insertion depth $(\mathrm{mm})$ & Resistance $(\mu \Omega)$ & insertion depth $(\mathrm{mm})$ & Resistance $(\mu \Omega)$ \\
\hline 55 & 16.256 & 55 & 16.019 \\
50 & 16.4395 & 50 & 17.439 \\
45 & 17.5568 & 45 & 18.556 \\
40 & 18.1748 & 40 & 19.174 \\
35 & 18.5363 & 35 & 22.536 \\
30 & 18.5511 & 30 & 24.551 \\
25 & 18.5826 & 25 & 27.582 \\
\hline
\end{tabular}

Table 3. Different test method of measuring resistance.

\begin{tabular}{cc}
\hline current(A) & Resistance $(\mu \Omega)$ \\
\hline 1000 & 256.521 \\
1500 & 256.897 \\
2000 & 257.034 \\
2500 & 257.436 \\
3000 & 257.635 \\
3500 & 257.873 \\
\hline
\end{tabular}




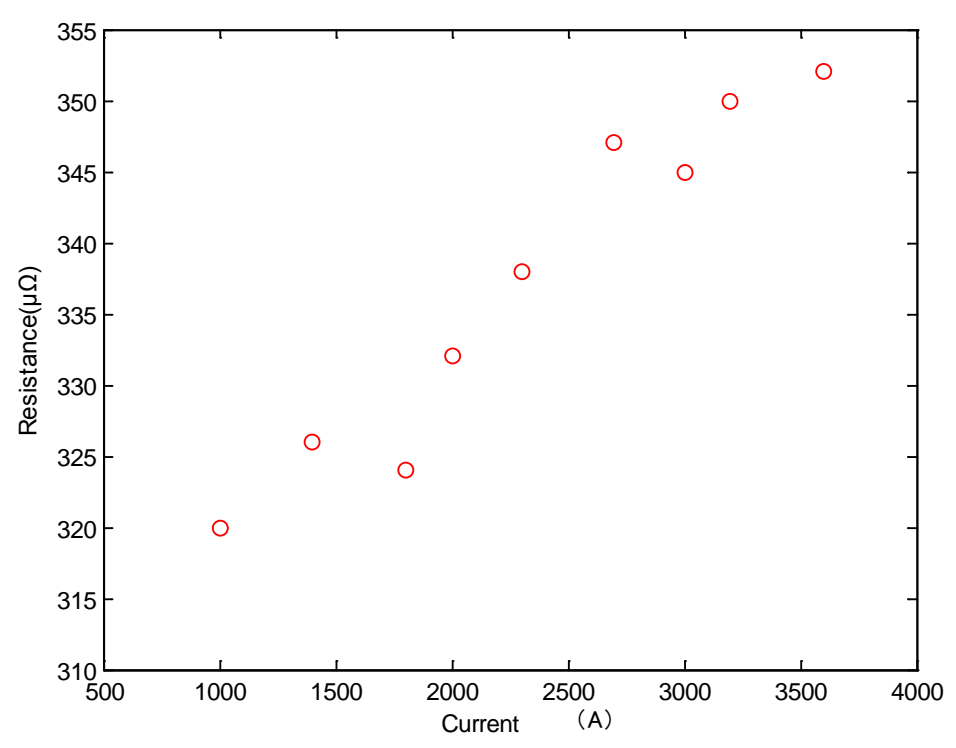

Figure 6. Resistances obtained when current were passed through GIS containing a poor contact.

Tests have also carried out on an 8-m-long laboratory mock up of a GIS conductor section consisting of two concentric aluminum tubes of 50 and $10 \mathrm{~cm}$ diameters. The total resistance was increased to approximately 300 $\mu \Omega$ by adding a short stainless steel section. A somewhat poor contact (around $40 \mu \Omega$ ) made by partially unscrewing the bolts was inserted in the circuit. Figure 6 shows resistance plotted as a function of current when 9 current pulses of varying amplitude were passed through this model simulating a GIS section with a poor contact. Resistances obtained when current pulses of different amplitudes were passed through a model of a GIS containing a poor contact. The resistance increases with increasing currents indicating that the contact spots become significantly heated at high currents.

\section{Conclusions}

The following conclusions can be drawn from this paper.

a) This method can not only provide excitation currents up to several kiloamperes, but also can make the device of portable. What's more, it can accurately measure the loop resistance of GIS conductor pole.

b) Through the simulation results proved that when GIS conductor pole contacts well, the variation of temperature is not obvious. However when GIS conductor pole contacts bad, the temperature is higher when the contact condition is worse.

c) Through experiment results proved that when GIS conductor pole has a good contact, two methods of testing can accurately measure resistance. However when GIS conductor pole has a poor contact, the method of impact current voltage drop can be used to detect contact degradation in GIS at an early stage.

\section{References}

[1] Runde, M., Lillevik, O. and Larsen, V. (2004) Condition Assessment of Contacts in Gas-Insulated Substations. IEEE Transactions on Power Delivery, 19, 609-617. http://dx.doi.org/10.1109/TPWRD.2003.822942

[2] Landry, M., Turcotte, O. and Brikci, F. (2008) A Complete Strategy for Conducting Dynamic Contact Resistance Measurements on HV Circuit Breakers. IEEE Transactions on Power Delivery, 23, 710-716. http://dx.doi.org/10.1109/TPWRD.2008.917694

[3] Zou, J.-Y., Zhang, L. and Song, J.-Y. (2004) Development of the 40 V Hybrid Super Capacitor Unit. The 12th Symposium on Electromagnetic Launch Technology, Snowbird, 25-28 May 2004, 197-201.

[4] Liu, S.Y. and Dougal, R.A. (2004) Design and Analysis of a Current-Mode Controlled Battery/Ultra-Capacitor Hybrid. Conference Record of the 2004 IEEE Industry Applications Conference, Seattle, 3-7 October 2004, 1140-1145.

[5] Haryono, V., Sirait, K.T., Tumiran and Berahim, H. (2008) The Computer Simulation for High Amplitude Impulse Current Design. International Conference on High Voltage Engineering and Application, 207-210. 
http://dx.doi.org/10.1109/ICHVE.2008.4773910

[6] Johal, H. (2008) Coil Current Analysis Method for Predictive Maintenance of Circuit Breakers. In: Transmission and Distribution Conference and Exposition.

[7] Runde, M. (1999) Vibration Analysis for Periodic Diagnostic Testing of Circuit-Breakers. 11th International Symposium on High Voltage Engineering, Conf. Publ. No. 467. http://dx.doi.org/10.1049/cp:19990517

[8] Meng, Y.P. (2006) The Detection of the Closing Moments of a Vacuum Circuit Breaker by Vibration Analysis. IEEE Transactions on Power Delivery, 21, 652-658. http://dx.doi.org/10.1109/TPWRD.2005.855475

[9] Landry, M. (2008) An Improved Vibration Analysis Algorithm as a Diagnostic Tool for Detecting Mechanical Anomalies on Power Circuit Breakers. IEEE Transactions on Power Delivery, 23, 1986-1994. http://dx.doi.org/10.1109/TPWRD.2008.2002846

[10] Monzel, C. and Henneberger, G. (2002) Temperature Solver for Highly Nonlinear Ferromagnetic Materials for Thin Moving Sheets in Transversal Flux Induction Heating. IEEE Transaction on Magnetics, 38, 937-940. http://dx.doi.org/10.1109/20.996241 